\title{
Augmented Lagrangian Method for Thin Plates with Signorini Boundaries
}

\author{
Erik Burman ${ }^{\star}$ Peter Hansbo ${ }^{\dagger}$, and Mats G. Larson ${ }^{\ddagger}$ \\ ^ Department of Mathematics, University College London, London, UK-WC1E 6BT, United \\ Kingdom \\ ${ }^{\dagger}$ Department of Mechanical Engineering, Jönköping University, SE-55111 Jönköping, Sweden \\ ${ }^{\ddagger}$ Department of Mathematics and Mathematical Statistics, Umeå University, SE-90187 \\ Umeå, Sweden
}

\begin{abstract}
We consider $C^{1}$-continuous approximations of the Kirchhoff plate problem in combination with a mesh dependent augmented Lagrangian method on a simply supported Signorini boundary.
\end{abstract}

\section{Introduction}

To introduce the augmented Lagrangian method we first consider a simple Poisson problem, find $u$ such that

$$
-\Delta u=f \text { in } \Omega, \quad u=g \text { on } \Gamma
$$

where $\Omega$ is a bounded domain with boundary $\Gamma:=\partial \Omega$ and exterior unit normal $n$,

The Lagrange multiplier approach to prescribing $u=g$ is to seek stationary points to

$$
\mathcal{L}(v, \mu):=\frac{1}{2} a(v, v)-\langle\mu, v-g\rangle_{\Gamma}-(f, v)
$$

where

$$
(f, v):=\int_{\Omega} f v d \Omega, a(u, v):=\int_{\Omega} \nabla u \cdot \nabla v d \Omega
$$

and $\langle\cdot, \cdot\rangle_{\Gamma}$ denotes the $H^{-1 / 2} / H^{1 / 2}$-duality pairing. Whenever the arguments are smooth enough we define,

$$
\langle\mu, v-g\rangle_{\Gamma}:=\int_{\Gamma} \mu(v-g) d s
$$

Stationary points are given by finding $(u, \lambda) \in H^{1}(\Omega) \times H^{-1 / 2}(\Gamma)$ such that

$$
a(u, v)-\langle\lambda, v\rangle_{\Gamma}=(f, v) \quad \forall v \in H^{1}(\Omega)
$$




$$
\langle\mu, u\rangle_{\Gamma}=\langle\mu, g\rangle_{\Gamma} \quad \forall \mu \in H^{-1 / 2}(\Gamma)
$$

Formally, the Lagrange multiplier is given by $\lambda=\partial_{n} u$, where $\partial_{n} v:=\boldsymbol{n} \cdot \nabla v$. In a discretization of this problem, the approximation of the multiplier and the displacement must fulfil an inf-sup condition ensuring that the problem will not be overconstrained.

We now augment the Lagrangian by a penalty term and seek stationary points to

$$
\mathcal{L}(v, \mu):=\frac{1}{2} a(v, v)-\langle\mu, v-g\rangle_{\Gamma}+\frac{1}{2}\left\|\gamma^{1 / 2}(v-g)\right\|_{\Gamma}^{2}-(f, v)
$$

leading to the problem of finding $(u, \lambda) \in H^{1}(\Omega) \times H^{-1 / 2}(\Gamma)$ such that

$$
a(u, v)-\langle\lambda, v\rangle_{\Gamma}+\langle\gamma u, v\rangle_{\Gamma}-\langle\mu, u\rangle_{\Gamma}=(f, v)+\langle\gamma g, v\rangle_{\Gamma}-\langle\mu, g\rangle_{\Gamma}
$$

for all $(v, \mu) \in H^{1}(\Omega) \times H^{-1 / 2}(\Gamma)$. The discretization of this problem requires the same careful balance between approximation spaces for the primal variable and the multiplier as does the standard Lagrange multiplier method. Indeed if we introduce the space

$$
V_{h}:=\left\{v_{h} \in H^{1}(\Omega):\left.v_{h}\right|_{K} \in \mathbb{P}_{k}(K), \forall K \in \mathcal{T}_{h}\right\}, \quad \text { for } k \geq 1
$$

where $\mathcal{T}_{h}$ is a conforming quasi-uniform partition of $\Omega$ and $\mathbb{P}_{k}(K)$ denotes the set of polynomials of degree less than or equal to $k$ on the element $K$ for the discretization of $u$, we must find a mulitplier space $\Lambda_{h}$ such that the inf-sup condition is satisfied. However, If we seek $u_{h} \in V_{h}$ with the discrete multiplier $\lambda_{h}:=\partial_{n} u_{h}$, we recover Nitsche's method:

$$
a\left(u_{h}, v\right)-\left\langle\partial_{n} u_{h}, v\right\rangle_{\Gamma}-\left\langle\partial_{n} v, u_{h}\right\rangle_{\Gamma}+\left\langle\gamma u_{h}, v\right\rangle_{\Gamma}=(f, v)+\left\langle g, \gamma v-\partial_{n} v\right\rangle_{\Gamma}
$$

for all $v \in V_{h}$, with $\mu=\partial_{n} v$, which is stable with the choice $\gamma=\gamma_{0} / h$, where $h$ is the local meshsize and $\gamma_{0}$ large enough.

If we alternatively consider a stable discretization $\lambda_{h} \in \Lambda_{h}$, the discrete problem can be seen as seeking stationary points $\left(u_{h}, \lambda_{h}\right) \in V_{h} \times \Lambda_{h}$ to the modified Lagrangian

$$
\mathcal{L}_{h}(v, \mu):=\frac{1}{2} a(v, v)+\frac{1}{2}\left\|\gamma^{1 / 2}\left(v-g-\gamma^{-1} \mu\right)\right\|_{\Gamma}^{2}-\left\|\gamma^{-1 / 2} \mu\right\|_{\Gamma}^{2}-(f, v)_{\Omega}
$$

which is obtained from $\mathcal{L}$ in $(7)$ by rearranging terms and noting that the discrete multiplier is in $L_{2}(\Gamma)$.

We now turn to an inequality constraint on the boundary: $u \leq g$ on $\Gamma$. The corresponding Kuhn-Tucker conditions read:

$$
u-g \leq 0, \quad \lambda \leq 0, \quad \lambda(u-g)=0 \quad \text { on } \Gamma .
$$

These conditions can alternatively be written (cf. [6])

$$
\lambda=-\gamma\left[u-g-\gamma^{-1} \lambda\right]_{+}
$$


where $\gamma \in \mathbb{R}^{+},[x]_{+}=\max (x, 0)$. We can now, following Alart and Curnier [1], define the following discrete augmented Lagrangian

$$
\mathcal{L}_{h}(v, \mu):=\frac{1}{2} a(v, v)+\frac{1}{2}\left\|\gamma^{1 / 2}\left[v-g-\gamma^{-1} \mu\right]_{+}\right\|_{\Gamma}^{2}-\left\|\gamma^{-1 / 2} \mu\right\|_{\Gamma}^{2}-(f, v)
$$

The corresponding Euler-Lagrange equations read: find $\left(u_{h}, \lambda_{h}\right) \in V_{h} \times \Lambda_{h}$ such that

$$
a\left(u_{h}, v\right)+\left\langle\gamma\left[u_{h}-g-\gamma^{-1} \lambda_{h}\right]_{+}, v\right\rangle_{\Gamma}=(f, v) \quad \forall v \in V_{h}
$$

and

$$
\left\langle\gamma\left[u_{h}-g-\gamma^{-1} \lambda_{h}\right]_{+}+\lambda_{h}, \gamma^{-1} \mu\right\rangle_{\Gamma}=0 \quad \forall \mu \in \Lambda_{h}
$$

If $\left[u_{h}-g-\gamma^{-1} \lambda_{h}\right]_{+}=0$ (no contact) then $\lambda_{h}=0$ and if $\left[u_{h}-g-\gamma^{-1} \lambda_{h}\right]_{+}>0$ (contact) we recover the standard augmented formulation for the imposition of the Dirichlet condition $u=g$. The multiplier approach (15)-(16) using a stable pair $V_{h} \times \Lambda_{h}$ was shown to produce approximations of optimal accuracy in [4]. Now set $\lambda_{h}=\partial_{n} u_{h}, \mu=\partial_{n} v$ and seek $u_{h} \in V_{h}$ such that

$$
a\left(u_{h}, v\right)+\left\langle\gamma\left[u_{h}-g-\gamma^{-1} \partial_{n} u_{h}\right]_{+}, v-\gamma^{-1} \partial_{n} v\right\rangle_{\Gamma}-\left\langle\gamma^{-1} \partial_{n} u_{h}, \partial_{n} v\right\rangle_{\Gamma}=(f, v)
$$

for all $v \in V_{h}$. With the choice $\gamma=\gamma_{0} / h$ this is the Nitsche method for Signorini problems first proposed in the context of elastic contact by Chouly and Hild [6]. For more information on augmented Lagrangian methods and variants thereof, see [3].

\section{The Kirchhoff plate model}

We now proceed formally to extend the discussion to the Kirchhoff plate model, posed on a domain $\Omega \subset \mathbb{R}^{2}$ with boundary $\Gamma=\partial \Omega$ and exterior unit normal $\boldsymbol{n}$. We seek an out-of-plane (scalar) displacement $u$ to which we associate the strain (curvature) tensor

$$
\kappa(u):=\varepsilon(\nabla u):=\frac{1}{2}(\nabla \otimes(\nabla u)+(\nabla u) \otimes \nabla)=\nabla \otimes \nabla u
$$

and the plate stress (moment) tensor

$$
\begin{aligned}
\boldsymbol{M}(u):=\boldsymbol{\sigma}(\nabla u): & =D\left(\varepsilon(\nabla u)+\nu(1-\nu)^{-1} \operatorname{div} \nabla u \boldsymbol{I}\right) \\
& =D\left(\boldsymbol{\kappa}(u)+\nu(1-\nu)^{-1} \Delta u \boldsymbol{I}\right)
\end{aligned}
$$

where

$$
D=\frac{E t^{3}}{12(1+\nu)}
$$

with $E$ the Young's modulus, $\nu$ the Poisson's ratio, and $t$ the plate thickness. We will use the standard convention that all quantities are positive downwards. 
The Kirchhoff equilibrium problem takes the form: given the out-of-plane load (per unit area) $f$, find the displacement $u$ such that

$$
\operatorname{div} \operatorname{div} M(u)=f \quad \text { in } \Omega
$$

where div and div denote the divergence of a tensor and a vector field, respectively. We shall first consider a smooth boundary $\Gamma$ with simply supported boundary conditions

$$
u=0 \quad \text { on } \Gamma, \quad M_{n n}(u)=0 \quad \text { on } \Gamma
$$

where $M_{a b}=\boldsymbol{a} \cdot \boldsymbol{M} \cdot \boldsymbol{b}$ for $\boldsymbol{a}, \boldsymbol{b} \in \mathbb{R}^{2}$. Defining the tangent vector on the boundary as $\boldsymbol{t}=\left(n_{2},-n_{1}\right)$, multiplying by a test function $v$ and using repeated integration by parts we find that

$$
\begin{aligned}
(\operatorname{div} \operatorname{div} \boldsymbol{M}(u), v)= & (\boldsymbol{M}(u), \boldsymbol{\kappa}(v))-\left\langle M_{n n}(u), \partial_{n} v\right\rangle_{\Gamma} \\
& -\left\langle M_{n t}(u), \partial_{t} v\right\rangle_{\Gamma}+\langle\boldsymbol{n} \cdot \operatorname{div} \boldsymbol{M}(u), v\rangle_{\Gamma}
\end{aligned}
$$

In the case of a smooth boundary we note that

$$
\left\langle M_{n t}(u), \partial_{t} v\right\rangle_{\Gamma}=-\left\langle\partial_{t} M_{n t}(u), v\right\rangle_{\Gamma}
$$

and by introducing the Kirchhoff shear force $T:=\boldsymbol{n} \cdot \operatorname{div} \boldsymbol{M}+\partial_{t} M_{n t}$ we have

$$
(\operatorname{div} \operatorname{div} \boldsymbol{M}(u), v)=(\boldsymbol{M}(u), \boldsymbol{\kappa}(v))-\left\langle M_{n n}(u), \partial_{n} v\right\rangle_{\Gamma}+\langle T(u), v\rangle_{\Gamma}
$$

Taking into account the boundary conditions, the variational problem thus takes the form: find

$$
u \in V=\left\{v \in H^{2}(\Omega): v=0 \text { on } \Gamma\right\}
$$

such that

$$
(\boldsymbol{M}(u), \boldsymbol{\kappa}(v))=(f, v) \quad \forall v \in V
$$

We will next consider the Signorini condition $u \geq g$ on $\Gamma$, which corresponds to a case where the plate boundary rests on a rigid foundation but is not fixed to it. Introducing a multiplier representing $T(u)$ we have that

$$
\begin{array}{rll}
\operatorname{div} \operatorname{div} \boldsymbol{M}(u)=f & & \text { in } \Omega \\
M_{n n}(u)=0 & & \text { on } \Gamma \\
T(u)+\lambda=0 & & \text { on } \Gamma \\
u-g \geq 0 & & \text { on } \Gamma \\
\lambda \leq 0 & & \text { on } \Gamma \\
\lambda(u-g)=0 & & \text { on } \Gamma
\end{array}
$$

In this case, the Kuhn-Tucker conditions can be rewritten

$$
\lambda=-\gamma\left[g-u-\gamma^{-1} \lambda\right]_{+}
$$


Remark 2.1 (Handling polygonal domains) In the case of a domain with piecewise smooth boundaries, so called Kirchhoff corner forces occur in corner points [9, Chapter 5.5]. This case was considered by Nazarov et al. [8] but with an alternative formulation (the biharmonic operator, leading to quite different boundary conditions). We here assume that $\Gamma$ consists of smooth connected parts $\Gamma_{i}$ with corner intersections at $\boldsymbol{x}_{i}$. Now 26) has to be modified as follows:

$$
\left\langle M_{n t}(u), \partial_{t} v\right\rangle_{\Gamma}=-\left\langle\partial_{t} M_{n t}(u), v\right\rangle_{\Gamma}+\sum_{i}\left(M_{n t}^{-}\left(u\left(\boldsymbol{x}_{i}\right)-M_{n t}^{+}\left(u\left(\boldsymbol{x}_{i}\right)\right)\right) v\left(\boldsymbol{x}_{i}\right)\right.
$$

where $M_{n t}^{ \pm}\left(u\left(\boldsymbol{x}_{i}\right)\right)=\lim _{\epsilon \downarrow 0} M_{n t}\left(u\left(x_{i} \pm \epsilon, y_{i} \pm \epsilon\right)\right)$, giving rise to (virtual work of) point forces in the corners. Unlike the Kirchhoff shear forces, the corner forces are present whether there is contact or not, and are implemented as contributions to the stiffness matrix.

\section{$3 \quad$ Finite element method}

We will use $C^{1}$-continuous element on meshes $\mathcal{K}_{h}$ made up of rectangles. On each element $K \in \mathcal{K}_{h}$ we let $Q_{3}$ denote the outer product of cubic polynomials:

$$
Q_{3}=\left\{p(x, y): p(x, y)=\sum_{0 \leq i, j \leq 3} c_{i j} x^{i} y^{j}\right\}
$$

where $c_{i j}$ are constants. The approximation space associated with the BognerFox-Schmit (BFS) element first proposed in 2 is defined by

$$
V_{h}=\left\{v \in C^{1}(\Omega):\left.v\right|_{K} \in Q_{3}, \forall K \in \mathcal{K}_{h}\right\}
$$

The shape functions on the BFS element are then made up of outer products of cubic splines, typically used for beam problems. We refer to Zhang [10 for further details on this approximation. Though this element might seem limited in view of it only being defined on rectangular meshes, the recent CutFEM for BFS [5] extends its use to arbitrary geometries.

In analogy with (17) we now pose the following discrete problem: find $\left(u_{h}, \lambda_{h}\right) \in V_{h} \times \Lambda_{h}, \Lambda_{h}$ to be chosen, such that

$$
\left(\boldsymbol{M}\left(u_{h}\right), \boldsymbol{\kappa}(v)\right)-\left\langle\gamma\left[g-u_{h}-\gamma^{-1} \lambda_{h}\right]_{+}, v\right\rangle_{\Gamma}=(f, v) \quad \forall v \in V_{h}
$$

and

$$
-\left\langle\gamma\left[g-u_{h}-\gamma^{-1} \lambda_{h}\right]_{+}, \gamma^{-1} \mu\right\rangle_{\Gamma}-\left\langle\gamma^{-1} \lambda_{h}, \mu\right\rangle_{\Gamma}=0 \quad \forall \mu \in \Lambda_{h}
$$

We next consider replacing $\lambda_{h}$ following the ideas of Section 1. To this end, we formally set $\lambda_{h}=-T\left(u_{h}\right)$ and $\mu=-T(v)$ to obtain the problem of finding $u_{h} \in V_{h}$ such that

$$
\left(\boldsymbol{M}\left(u_{h}\right), \boldsymbol{\kappa}(v)\right)-\left\langle\gamma\left[g-\psi\left(u_{h}\right)\right]_{+}, \psi(v)\right\rangle_{\Gamma}-\left\langle\gamma^{-1} T\left(u_{h}\right), T(v)\right\rangle_{\Gamma}=(f, v)
$$

for all $v \in V_{h}$, where $\psi(w):=w-\gamma^{-1} T(w)$. Setting now $\gamma=\gamma_{0} / h^{3}$ stability, existence and uniqueness of discrete solution can be shown combining the results of [7] and [4. We leave the details to a forthcoming publication. 


\section{Numerical results}

We consider a quadratic plate $(0,1) \times(0,1)$ of thickness $t=0.1$ and with moduli of elasticity $E=100, \nu=0.5$. This plate is loaded by a point force of unit strength. The free parameter was chosen as $\gamma_{1}=10^{4} \mathrm{D}$. The maximum displacement on the boundary is set to $g=0$.

\subsection{Point load in the center of the plate}

We load the plate with a unit point load at the center. In Fig. 1 we show the computed displacement field with the Signorini boundary indicated by a dotted line. In Fig 2 we show the computed Kirchhoff shear force in the contact zone (evaluated at the midpoint of each element side) on a sequence of uniformly refined meshes. We note the symmetry of the solution.

\subsection{Point load at $(3 / 4,3 / 4)$}

The same plate is now loaded with unit point load at $(3 / 4,3 / 4)$. In Fig. 3 we show the computed displacement field, again with the Signorini boundary indicated by a dotted line. In Fig 4 we show the corresponding Kirchhoff shear force in the contact zone. We note the elevation of the shear force close to the first point of contact, similar, but more pronounced, to Fig. 2

\section{Acknowledgments}

This research was supported in part by the Swedish Foundation for Strategic Research Grant No. AM13-0029, the Swedish Research Council Grants Nos. 2017-03911 and 2018-05262, and the Swedish Research Programme Essence. Erik Burman was partially supported by the grant: EP/P01576X/1.

\section{References}

[1] P. Alart and A. Curnier. A mixed formulation for frictional contact problems prone to Newton like solution methods. Comput. Methods Appl. Mech. Engrg., 92(3):353-375, 1991.

[2] F. K. Bogner, R. L. Fox, and L. A. Schmit. The generation of interelement compatible stiffness and mass matrices by the use of interpolation formulae. In Proc. Conf. Matrix Methods in Struct. Mech., AirForce Inst. of Tech., Wright Patterson AF Base, Ohio, pages 397-444, 1965.

[3] E. Burman and P. Hansbo. Deriving robust unfitted finite element methods from augmented Lagrangian formulations. In Geometrically unfitted finite element methods and applications, volume 121 of Lect. Notes Comput. Sci. Eng., pages 1-24. Springer, Cham, 2017. 
[4] E. Burman, P. Hansbo, and M. G. Larson. Augmented Lagrangian finite element methods for contact problems. ESAIM Math. Model. Numer. Anal., 53(1):173-195, 2019.

[5] E. Burman, M. G. Larson, and P. Hansbo. Cut Bogner-Fox-Schmit elements for plates. arXiv 1911.00239, 2019.

[6] F. Chouly and P. Hild. A Nitsche-based method for unilateral contact problems: numerical analysis. SIAM J. Numer. Anal., 51(2):1295-1307, 2013.

[7] P. Hansbo and M. G. Larson. A discontinuous Galerkin method for the plate equation. Calcolo, 39(1):41-59, 2002.

[8] S. A. Nazarov, A. Stylianou, and G. Sweers. Hinged and supported plates with corners. Z. Angew. Math. Phys., 63(5):929-960, 2012.

[9] V. Slivker. Mechanics of structural elements: theory and applications. Springer Science \& Business Media, 2006.

[10] S. Zhang. On the full $C_{1}-Q_{k}$ finite element spaces on rectangles and cuboids. Adv. Appl. Math. Mech., 2(6):701-721, 2010. 


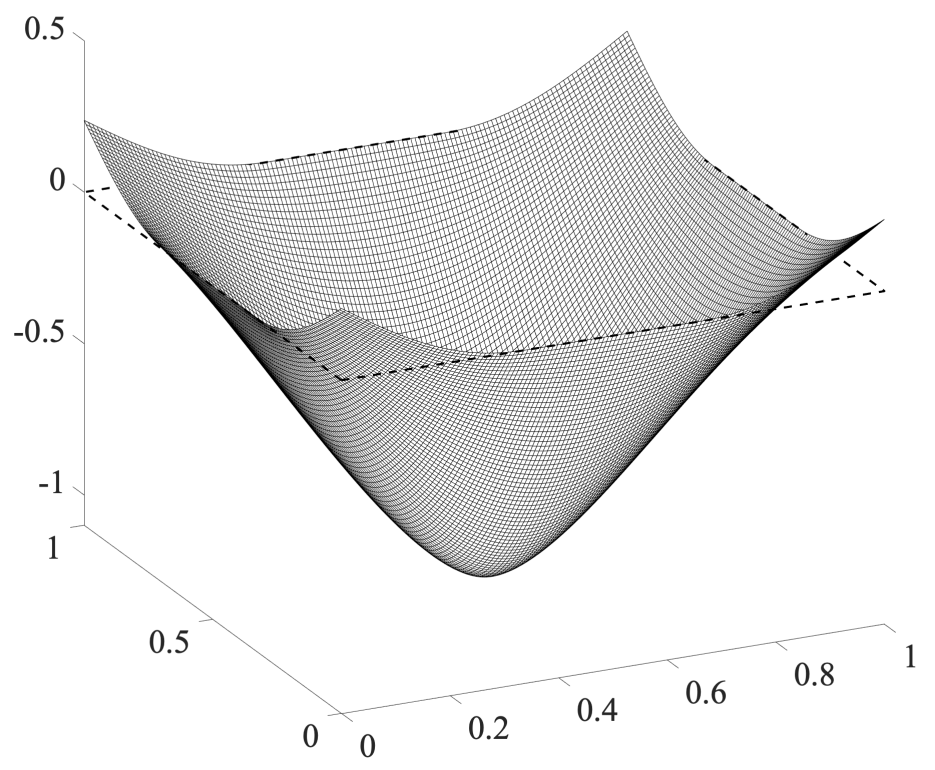

Figure 1: Elevation of the solution on the finest mesh in a sequence. Point load at $(1 / 2,1 / 2)$. 

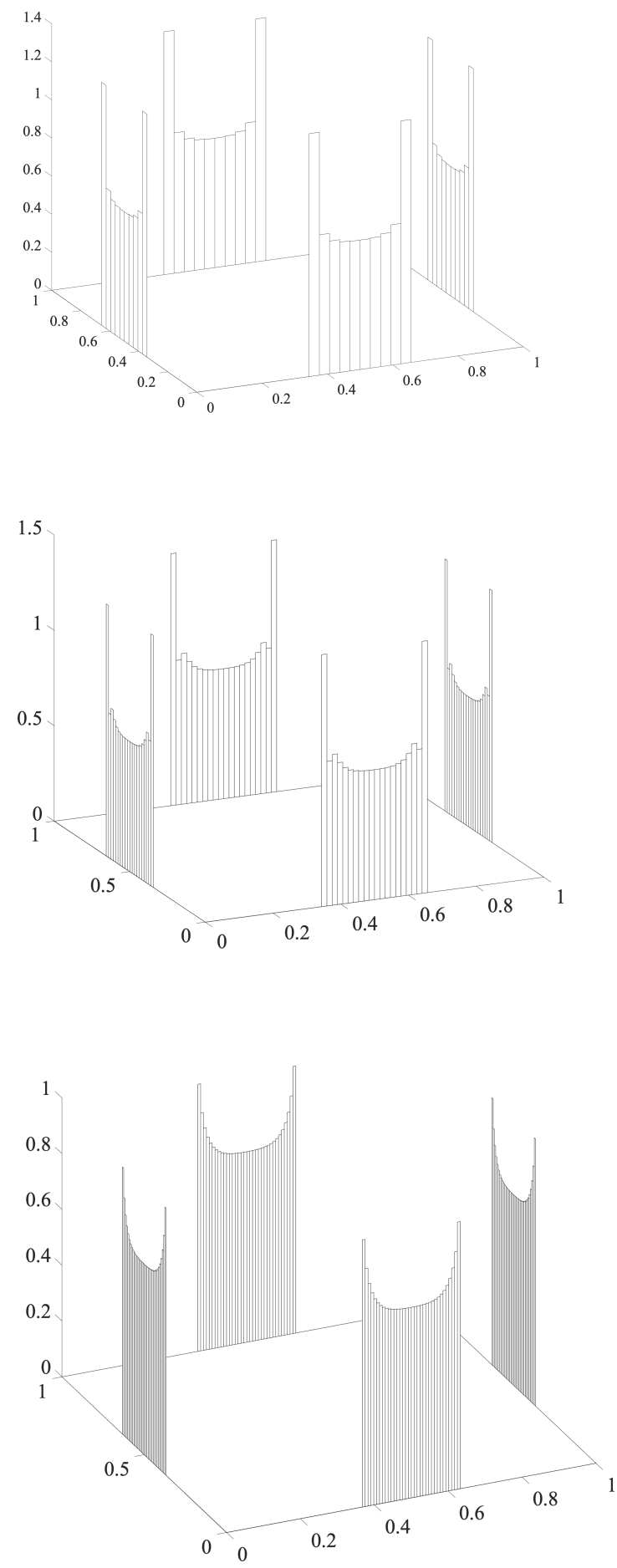

9

Figure 2: Kirchhoff shear forces in the contact zone on consecutively refined meshes. 


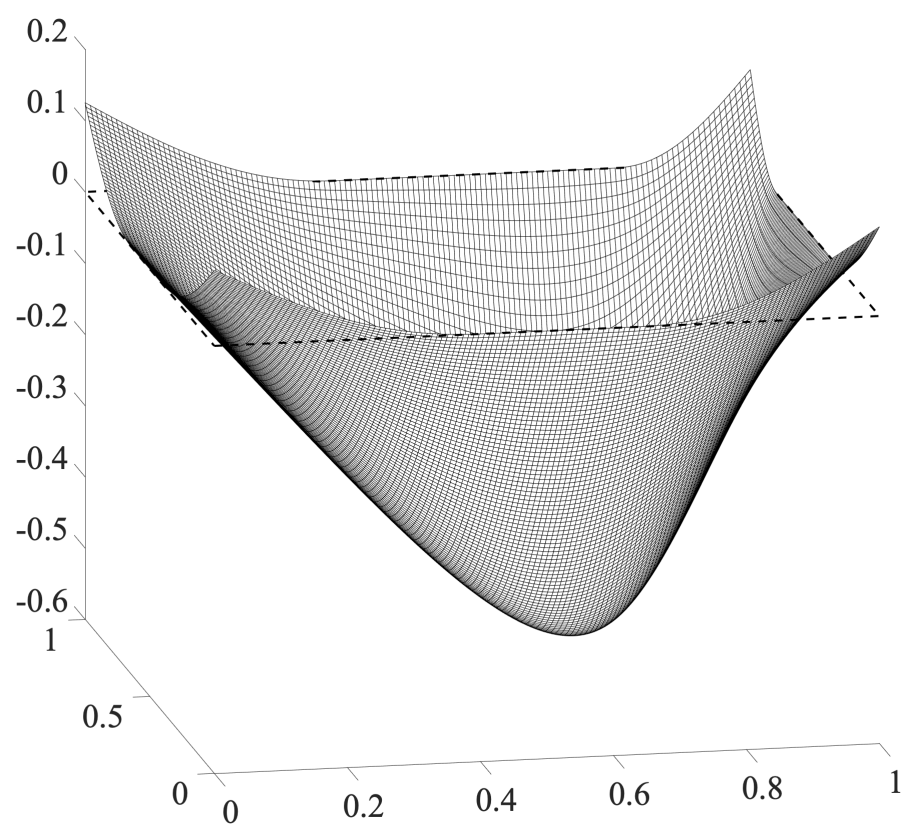

Figure 3: Elevation of the solution on the finest mesh in a sequence. Point load at $(3 / 4,3 / 4)$. 

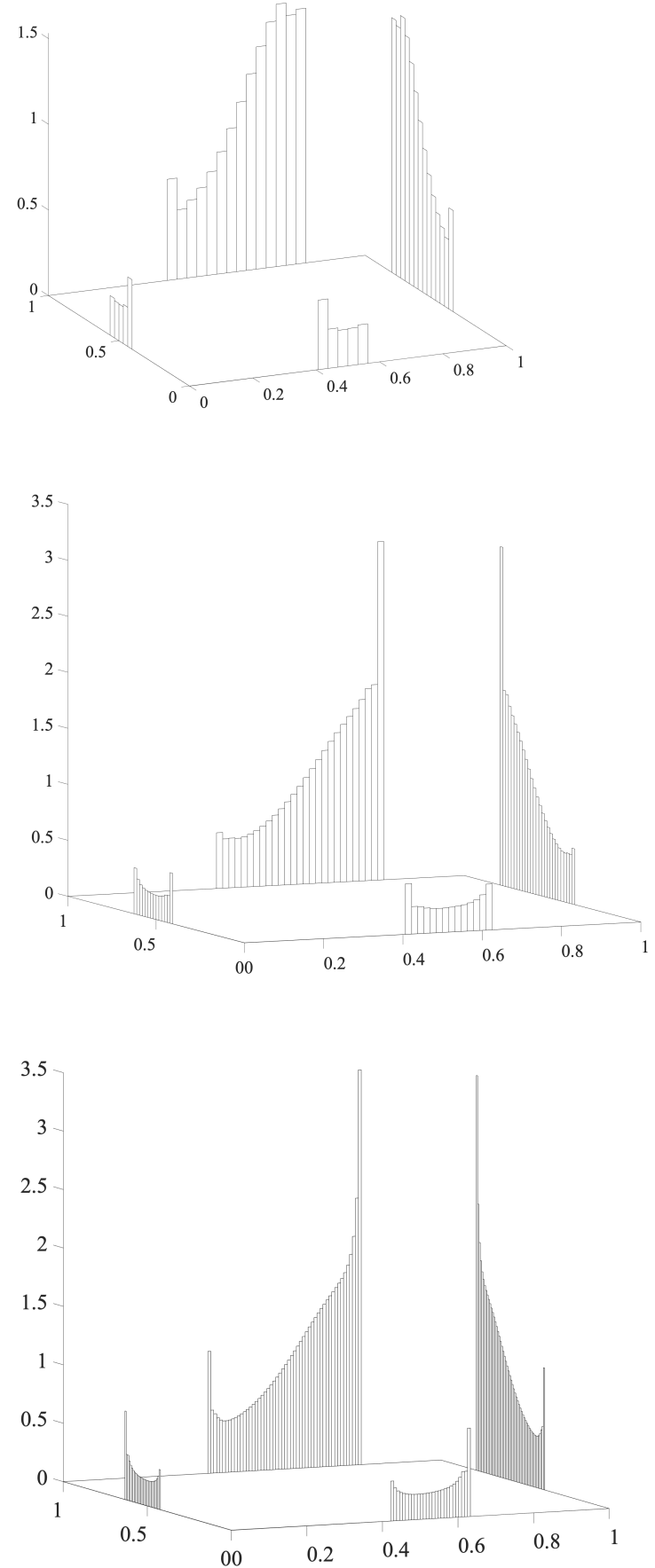

Figure 4: Kirchhoff shear forces in the contact zone on consecutively refined meshes. 